\title{
Synthesis of New inhibitors of the FibrillogenesisAß42: Alzheimer's Disease
}

Type of article: Conference abstract

\author{
Assia Keniche1,2, katiaouled Taleb2, Joseph Kajima Mulengi3. \\ 1.PhD of Bioorganic and therapeutic chemistry, Maghnia Center University, Tlemcen, Algeria. \\ 2. Laboratory of Organic Chemistry Natural Products and Analysis University of Tlemcen, Algeria. \\ 3.Faculté des sciences, University of Tlemcen, Algeria. \\ e-mail: keniche_assia@yahoo.fr
}

\begin{abstract}
:
Background: The Alzheimer's disease (AD) is a devastating neurodegenerative disorder and the most common cause of dementia. The pathological hallmarks of $\mathrm{AD}$ are the presence of neurofibrillary tangles and amyloid deposits in the brain of the patient, as already defined by Alois Alzheimer in 1907. The aim of our study is to inhibit the formation the folding of amyloid protein by new synthesis of $\beta$-sheet breaker peptides.

Methods: Our approach consist to develop $\beta$-sheet breaker peptides as a novel strategy to inhibit and reverse amyloidosis in AD. The synthesis was down by peptid coupling method.

Results: Several $\beta$-sheet breaker peptides have been designed based on the hypothesis that amyloid deposition could be inhibited by short synthetic peptides partially homologous to the $A \beta$ region undergoing conformational changes to give rise to the amyloidogenic $A \beta$ intermediate and containing residues disrupting as aziridine and CD for the $\beta$-sheet formation university Tlemcen 2017 ..

Conclusion: At present, there is no ideal drug for the treatment of $\mathrm{AD}$, and the searches for $\mathrm{AD}$ drugs remain an urgent issue in the pharmaceutical community. Due to its complex pathogenesis, a single target drug cannot cure this disease fundamentally. Dual or multiple target drugs involved in two or more aspects of AD pathogenesis may generate a synergistic effect and ultimately achieve an ideal therapeutic effect.
\end{abstract}

Keyword: Alzheimer, FibrillogenesisAß42, $\beta$-sheet.

\section{Conflict of interest}

This article is an abstract selected from the International Congress on Health Sciences and Medical Technologies, Algiers, Algeria, October 21-23, 2018.

\section{Authors' biography}

No biography

\section{References}

No references 